$\Rightarrow$ GYNAECOLOGICAL CANCER

\title{
SLN staging for endometrial cancer
}

Women with endometrial cancer who are found to have lymph-node-positive disease during surgical staging by lymphadenectomy benefit from adjuvant chemotherapy; however, the remaining $78 \%$ of women who have node-negative disease do not. Moreover, lymphadenectomy can have adverse effects, including lymphoedema and nerve damage. New data indicate that sentinel lymph-node (SLN)-based staging is a reliable alternative.

In the FIRES prospective cohort study, the diagnostic accuracy of SLN biopsy guided by fluorescence mapping with indocyanine green dye for the detection of metastases was compared with that of 'gold standard' pelvic \pm para-aortic lymphadenectomy. For generalizability, the FIRES study involved 18 surgeons across 10 centres (tertiary academic or community non-academic).

A total of 340 women underwent lymphadenectomy. Among the 293 patients with at least one SLN successfully mapped, 35 of 36 women with node-positive disease were correctly identified via SLN biopsy, equating to a sensitivity of $97.2 \%$ and negative predictive value of $99.6 \%$. Of the 47 patients without a SLN detected through fluorescence mapping, five had node-positive disease; patients with failed mapping would require full staging lymphadenectomy. Emma Rossi, lead author, opines: "the most important finding was the high sensitivity, with a confidence interval narrow enough to ensure a true sensitivity of no lower than $85 \%$. SLN biopsy also enabled the detection of isolated para-aortic metastases, albeit a rare event."

These results indicate that women with endometrial cancer can be safely offered a less-morbid staging procedure with comparable accuracy, thus enabling reliable risk assignment and therapeutic decision-making. As Rossi explains, however, "there is a commonly held belief that outcomes of patients, particularly those with high-grade cancers, are better after surgical removal of large numbers of lymph nodes." She concludes, "a phase III trial is, therefore, needed to compare staging with SLN biopsy versus lymphadenectomy in terms of oncological outcomes, such as disease recurrence and survival."

David Killock

ORIGINAL ARTICLE Rossi, E. C. et al. A comparison of sentinel lymph node biopsy to lymphadenectomy for endometrial cancer staging (FIRES trial): a multicentre, prospective, cohort study. Lancet Oncol. http://dx.doi.org/ 10.1016/S1470-2045(17)30068-2 (2017) 